\title{
U⿱宀⿻三丨口
}

\section{Communication Provision for a Team of Remotely Searching UAVs: A Mobile Relay Approach}

Luo, C., Ward, P., Cameron, S., Parr, G., \& McClean, S. (2012). Communication Provision for a Team of Remotely Searching UAVs: A Mobile Relay Approach. In Unknown Host Publication IEEE Communications Society.

Link to publication record in Ulster University Research Portal

\section{Published in:}

Unknown Host Publication

Publication Status:

Published (in print/issue): 03/12/2012

\section{Document Version \\ Author Accepted version}

\section{General rights}

Copyright for the publications made accessible via Ulster University's Research Portal is retained by the author(s) and / or other copyright owners and it is a condition of accessing these publications that users recognise and abide by the legal requirements associated with these rights.

\section{Take down policy}

The Research Portal is Ulster University's institutional repository that provides access to Ulster's research outputs. Every effort has been made to ensure that content in the Research Portal does not infringe any person's rights, or applicable UK laws. If you discover content in the Research Portal that you believe breaches copyright or violates any law, please contact pure-support@ulster.ac.uk. 


\title{
Communication Provision for a Team of Remotely Searching UAVs: A Mobile Relay Approach
}

\author{
Chunbo Luo ${ }^{\dagger}$, Paul Ward ${ }^{\ddagger}$, Stephen Cameron ${ }^{\ddagger}$, Gerard Parr ${ }^{\dagger}$, Sally McClean ${ }^{\dagger}$ \\ $\dagger$ School of Computing and Information Engineering, University of Ulster, Coleraine, BT52 1SA, UK \\ $\ddagger$ Oxford University Computer Science Department, Wolfson Building, Parks Road, Oxford OX1 3QD, UK. \\ E-mail: $\{c . l u o$, gp.parr, si.mcclean\}@ulster.ac.uk, \\ \{Paul.Ward, Stephen.Cameron\}@cs.ox.ac.uk
}

\begin{abstract}
In many applications it is desirable to deploy unmanned autonomous vehicles to remote real-world locations, at considerable distance from any fixed infrastructure, rendering direct communication impossible; for example in the Wilderness Search and Rescue (WiSaR) scenario. However, data transfer between such vehicles and other participants is required for control, safety, monitoring progress and sharing of acquired information. This paper proposes a method of using an Unmanned Aerial Vehicle (UAV) as a highly mobile relay, providing a delay tolerant link between a fixed base-station and a team of searching UAVs to meet this vital communication need. A communication model is described that permits a single relay to simultaneously collect data from multiple operational UAVs at pre-arranged meetings, both theoretical and experimental simulation results demonstrate the characteristics and effectiveness of this approach in a realistic cooperative sensing scenario.

Index Terms-UAV, Wilderness Search and Rescue, wireless
\end{abstract} communications, relay model

\section{INTRODUCTION}

In the Wilderness Search and Rescue (WiSaR) problem, the primary goal is to locate a missing person in a natural environment [1]. A promising approach is the deployment of a group of small rotor-craft Unmanned Aerial Vehicles (UAVs) to aid traditional human response teams [2]. The high mobility of such platforms makes them ideally suited to operating in large physical environments. In WiSaR scenarios, communication between all agents (base-station and UAVs) is vital to both achieve effective coordination and allow important collected data (images and video) to be made available to human rescue personnel. This paper addresses the problem of providing communication capability for a team of UAVs operating in a location remote to the base-station.

The primary difficulty of this problem arises from deploying physical robots into challenging real-world environments, producing a significant amount of uncertainty [3]. A common solution to this topic seeks to maintain a continuous link between operational UAVs and a base station in order to have low latency transfer of new information, for example [4]-[6]. However, it greatly limits the spatial range of these highly mobile vehicles, thus significantly restricting their deployment environment, scope and exploration freedom. Some proposed solutions position UAVs to form multi-hop links [4], [7], [8];

This work is funded by the EPSRC SUAAVE (Sensing Unmanned Autonomous Aerial Vehicles) research project (grant number: EP/F064179/1). this compromises the data gathering rate of a finite number of UAVs by placing them at certain geographical positions for the purpose of communication rather than data collection.

Recent work in distributed coordination, control and search strategies for UAVs provide methods to achieve a greater level of autonomy and do not require a strict permanent link to the base station. In this paper, we propose a communication model that allows data collected by a variable sized team of autonomous UAVs to efficiently transported to a base station without a continuous link. This enables searching UAVs to explore areas distant from the base station with greater freedom, thus increasing the spatial coverage and search rate, helping to minimize the delay in locating missing persons.

Specifically, a specialized UAV is designed to act as a combined relay and data mule for the UAV search team. Using its high degree of mobility this relayer travels between the base-station and distant location of the UAVs providing critical communication provision. At a pre-arranged meeting, all members of the team exchange data with the relay which carries multiple antennas (the number of searchers is less than or equal to the number of antennas and in this paper we use a maximum of 4 searchers) to avoid contention and minimize the time required for data exchange. The primary motivation of this method is to increase overall performance of the entire system, reducing the delay in locating missing persons. By relaxing the continuous connectivity constraint the approach aims to achieve improved utilization of the available UAVs.

\section{A. UAV Team Behaviour}

A search team consists of multiple UAVs searching a given region by passing their ground-ward facing sensors over the terrain. The UAVs behave in a coordinated manner to have a high rate of search and reduce redundant coverage (visiting the same area multiple times). Using the dual concepts of frontierbased search and pre-arranged rendezvous meetings the team aims to perform an efficient sensing task. The team behaviour takes inspiration from the original frontier exploration method [9], paired searcher/relay approaches to disaster response using mobile ground-base robots [10] and information sharing at prearranged meetings [11].

Each searching UAV maintains an individual map of the entire area, representing its current view of search progress (both visited and unvisited). When the UAV requires a new 
region to search it creates a list of potential frontiers in unvisited space using a region filling approach on this map that can be searched without changing altitude; a greedy heuristic selection procedure then chooses the next frontier to search. Using a sweep pattern the UAV then begins to pass its sensor over the designated region. To enable coordination of the search, each UAV periodically broadcasts its current location and a bounding box describing its search region to nearby team members with a short message. This allows the frontier selection process to discount any potential frontiers that either intersect with the operating area of another team member or could be searched with lower cost by another UAV.

At periodic pre-arranged meetings, termed rendezvous, all members of the team move toward a designated location to exchange information. This provides an opportunity to share representations of the search progress (by combining maps) and important sensory data gathered, such as images that may contain targets. These meetings include all UAVs, searchers and relay. Future rendezvous are scheduled at the previous occurrences, with the length of time between them determined by the relay's estimated return trip to base.

\section{Problem Definition And Model Description}

\section{A. Problem Definition}

Based on the UAV team behaviour described in Section I-A, the overall system communication requirements can be summarized as

1) A short term high bandwidth link between relay and searching UAVs for payload data exchange;

2) Delay tolerant link between the base and remotely situated UAVs.

The primary focus of this paper is to propose a communication model for the relay to efficiently collect acquired payload data from searching UAVs. This is essential for the system design to function as a whole and successfully perform the desired search behaviour. The second communication requirement is met by the general behaviour of the relay, exploiting high mobility of the platform to ferry data between a remote location and base, where it can inform the critical actions of human rescuers.

In addition, the communication model should exhibit the following desirable properties,

- Flexible number of access nodes; allowing the system to adapt online to changes in team size.

- High data rate within given bandwidth; modern sensing devices produce large volumes of data despite compression methods.

- Reliable connections; data loss can reduce efficiency as sensing must be repeated and at worst result in failure of the task.

- Optimize for team performance; the model should be configurable to allow maximum performance of the team through coordination of communications during rendezvous.
Throughout this paper, $\mathrm{T}$ and $\mathrm{r}$ denotes the transpose and conjugate transpose respectively. $\operatorname{tr}\{\cdot\}, \operatorname{det}\{\cdot\}$ and $E\{\cdot\}$ are the trace, determinant and expectation of a matrix respectively. log is the logarithm function with base 2 .

\section{B. System Model}

The system model is illustrated in Fig.1. We focus on the scenario where all UAVs are located at the required rendezvous and they all have data to transmit to the relay. All connections are constantly maintained, thus there is no bias towards any particular UAV. This assumption is only for the convenience of the theoretical analysis.

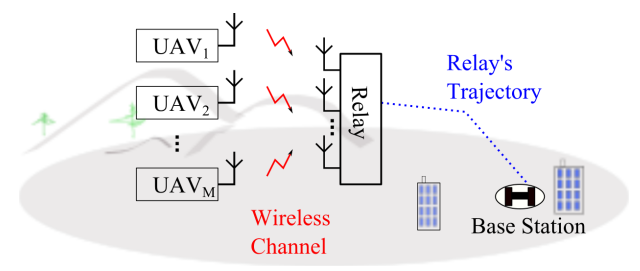

Fig. 1. The system model; comprising multiple searching UAVs, single relay UAV and base-station.

We denote the number of UAVs as $M$, each is equipped with one antenna and the relay has $N$ antennas $(N \geq M)$. All UAVs are simultaneously connected to the relay, thus creating spatial multiple access channels. We assume the channels suffer from slow fading and the relay has this channel information, obtained through channel estimation. At the $n$th time stamp, the received signal at relay $r$ is given by

$$
\mathbf{y}_{r}(n)=\mathbf{H}(n) \mathbf{V}(n) \mathbf{x}(n)+\mathbf{w}(n),
$$

where $\mathbf{H}(n)$ is the channel matrix, $\mathbf{V}(n)=$ $\left\{v_{1}(n), \ldots, v_{M}(n)\right\}$ is the precoding matrix of the multiple users, $\mathbf{x}(n)=\left[x_{1}, \ldots, x_{M}\right]^{\mathrm{T}}$ is the signal vector from the $M$ UAVs (Denoted as $U_{m}, \quad m=1,2, \ldots, M$ ) with $\mathrm{E}\left\{\mathbf{x}(n) \mathbf{x}(n)^{\mathrm{H}}\right\}=\operatorname{diag}\left\{P_{1}, \ldots, P_{M}\right\}$ and $\mathbf{w}(n)$ is additive Gaussian white noise (AWGN) with $\mathrm{E}\left\{\mathbf{w}(n) \mathbf{w}(n)^{\mathrm{H}}\right\}=N_{0}^{2} \mathbf{I}_{M} . P_{m}$ and $N_{0}$ are the corresponding symbol and noise variance. For the simplicity of expression, the time stamp $n$ is neglected for the remainder of this paper.

From (1), we can see an equivalence to a multiple input multiple output (MIMO) channel [12] except the signals from different antenna cannot be directly coordinated. Thus the decoding strategy can be performed by sequential interference cancellation (SIC) based minimum mean square (MMSE) algorithm. The detail of this method can be found in [13].

The precoding matrix $\mathbf{V}$ is particularly important in this flexible model as it implies a mapping and control strategy from the UAVs to the relay. This enables the proposed model to be configured to satisfy the immediate requirements, e.g. coordinating the transmission or improving the throughput of the desired UAVs. In this instance, we focus on minimizing the rendezvous period through coordinated transmission so that the UAVs can maximize their search time and the relay can exchange data with the base station more frequently, thus 
increasing efficiency of the whole system. The optimization of which will be discussed in the next section.

\section{Theoretical ANALYSis}

To investigate the properties, advantages and limitations of the proposed communication model a theoretical analysis is presented, giving further insights of how effective UAV cooperative networks should be built. Characteristics including mutual information and average data rate are evaluated using information metrics.

\section{A. Channel Model}

In this theoretical analysis, channels are modelled as independent and identical distribution (IID) Rician distributions [12], e.g. $\mathbf{H}_{i, j} \sim \mathcal{C N}\left(m, \sigma^{2}\right)$. Due to the targeted deployment environment and team operation the wireless channel contains both the line-of-sight (LOS) and non-LOS (NLOS) signals. A Rician distribution is used to model such channels, as follows,

$$
\mathbf{H}=\sqrt{\frac{K}{K+1}} \overline{\mathbf{H}}+\sqrt{\frac{1}{K+1}} \tilde{\mathbf{H}},
$$

where $K$ is the Rician factor, $\overline{\mathbf{H}}$ represents the LOS components and $\tilde{\mathbf{H}}$ denotes the NLOS components, which can be modeled as zero-mean complex Gaussian random variables with unit variance [14]. Since $\overline{\mathbf{H}}$ is deterministic, its trace can be obtained as $\operatorname{TR}\left\{\overline{\mathbf{H}} \overline{\mathbf{H}}^{\mathrm{H}}\right\}=M N$.

\section{B. Mutual Information}

The model shown in (1) has mutual information of

$$
\mathcal{I}=\log \operatorname{det}\left\{\mathbf{I}_{N}+\frac{1}{N_{0}} \mathbf{H W H}^{\mathrm{H}}\right\},
$$

where $\mathbf{I}_{N}$ is an $N \times N$ identity matrix and

$$
\mathbf{W}=\mathbf{V} \cdot \operatorname{diag}\left\{P_{1}, \ldots, P_{M}\right\} \cdot \mathbf{V}^{\mathrm{H}}=\operatorname{diag}\left\{P_{1} v_{1}^{2}, \ldots, P_{M} v_{M}^{2}\right\}
$$

Given the relation $\operatorname{det}\left(\mathbf{I}_{M}+\mathbf{A}_{M \times N} \mathbf{B}_{N \times M}\right)=\operatorname{det}\left(\mathbf{I}_{N}+\right.$ $\left.\mathbf{B}_{N \times M} \mathbf{A}_{M \times N}\right)$, the above equation can be transformed to

$$
\mathcal{I}=\log \operatorname{det}\left\{\mathbf{I}_{N}+\frac{1}{N_{0}} \mathbf{W} \mathbf{H}^{\mathrm{H}} \mathbf{H}\right\} .
$$

Furthermore, by introducing the eigenvalues of $\mathbf{H}^{\mathrm{H}} \mathbf{H}$, the mutual information given above can be expressed,

$$
\mathcal{I}=\log \left\{\prod_{m=1}^{M}\left(1+\frac{P_{m} v_{m}^{2}}{N_{0}} \lambda_{m}\right)\right\}
$$

Since the channel $\mathbf{H}$ suffers from slow fading, from (6), the target data rate of each individual can be adjusted to satisfy the required throughput.

\section{Average Data Rate}

The average data rate reveals the throughput of a model and is commonly used to verify system performance. For this model it can be written as

$$
\mathcal{E}=\mathrm{E}_{\mathbf{H}}\{\mathcal{I}\}=\mathrm{E}_{\mathbf{H}}\left\{\log \operatorname{det}\left(\mathbf{I}_{M}+\frac{1}{N_{0}} \mathbf{H} \mathbf{W} \mathbf{H}^{\mathrm{H}}\right)\right\}
$$

Since sequential interference cancellation based MMSE is used in this paper, the average data rate can be modified to,

$$
\mathcal{E}=\mathrm{E}\left\{\sum_{m=1}^{M} \log \left(1+\frac{P_{m} v_{m}^{2} \lambda_{m}}{N_{0}}\right)\right\} .
$$

For which the closed form can be written,

$$
\begin{aligned}
\mathcal{E}= & \int_{0 \leq \lambda_{m_{1}} \leq \ldots \leq \lambda_{m_{M}}}\left(\sum_{m=1}^{M} \log \left(1+\frac{P_{m} v_{m}^{2} \lambda_{m}}{N_{0}}\right)\right) \\
& \cdot f\left(\lambda_{m_{1}}, \ldots, \lambda_{m_{M}}\right) \mathrm{d} \lambda_{m_{1}} \ldots \mathrm{d} \lambda_{m_{M}} .
\end{aligned}
$$

The joint probability density function of sorted eigenvalues: $0 \leq \lambda_{m_{1}} \leq \lambda_{m_{2}} \leq \ldots \leq \lambda_{m_{M}}$ (where $\left.m_{i} \in\{1, \ldots, M\}\right)$ can be found in [15], is given by

$$
\begin{aligned}
f( & \left.\lambda_{m_{1}}, \ldots, \lambda_{m_{M}}\right) \\
& =C_{1} K^{-\bar{M}}(K+1)^{-\bar{M}+M N} \prod_{k<l}^{M}\left(\phi_{k}-\phi_{l}\right)^{-1} \\
& \cdot \prod_{k=1}^{M} \lambda_{k}^{N-M} \prod_{k<l}^{M}\left(\lambda_{k}-\lambda_{l}\right)^{-1} e^{-K \sum_{l} \phi_{l}} e^{-\sum_{l}(K+1) \lambda_{l}} \\
& \cdot \operatorname{det}\left\{{ }_{0} F_{1}\left(N-M+1, K(K+1) \phi_{k} \lambda_{l}\right)\right\},
\end{aligned}
$$

where $\phi_{1}, \phi_{2}, \ldots, \phi_{M}$ are the $M$ eigenvalues of $\overline{\mathbf{H}} \overline{\mathbf{H}}^{\mathrm{H}}, C_{1}$ is a normalizing constant, $\bar{M}=M(M-1) / 2$ and ${ }_{0} F_{1}(\cdot, \cdot)$ is a hypergeometric function [16] with scalar arguments given by

$$
{ }_{0} F_{1}(N-M+1, x)=(N-M) ! x^{\frac{N-M}{2}} I_{N-M}(2 \sqrt{x})
$$

and $I_{N-M}(\cdot)$ is the modified Bessel function of the first kind.

Denote $\prod_{k<l}^{M}\left(\lambda_{k}-\lambda_{l}\right)^{-1}$ as $\operatorname{det}^{-1}(\Lambda)$, and after several mathematical manipulations, the average data rate can be shown as

$$
\mathcal{E}=\frac{e^{-\Sigma_{m=1}^{M} \lambda_{m}} \operatorname{det}^{-1}(\Lambda)}{\ln (2)(\Gamma(N-M+1))^{M}} \sum_{m=1}^{M} \operatorname{det}(\Psi(m)),
$$

where $\Gamma(\cdot)$ is the gamma function and $\Psi(\cdot)$ is a $M \times M$ matrix with entries

$$
\{\Psi(k)\}_{i, j}= \begin{cases}\int_{0}^{\infty} y^{N-i} \ln (1+\rho y) e^{-y} & j=k \\ \times_{0} F_{1}\left(N-M+1, y \lambda_{j}\right) \mathrm{d} y, & \\ \Gamma(N-i+1) \times & \\ { }_{1} F_{1}\left(N-i+1, N-M+1, \lambda_{j}\right), & j \neq k\end{cases}
$$

and $\rho=P_{m} v_{m}^{2} / N_{0}$ is the normalized transmitter signal-tonoise ratio (SNR).

At a high SNR region, we can obtain the approximation of average data rate using Jensen's inequality, as follows,

$$
\mathcal{E} \leq \sum_{m=1}^{M} \log \left(1+\rho \mathrm{E}\left\{\lambda_{m}\right\}\right)
$$

The above equation provides a tight upper bound due to the nature of the logarithmic function with a large input value.

In addition to the system throughput described by the average data rate, we would also like to know the capacity 
region of the $M-\mathrm{UAV}$ channel, which is given as

$$
\begin{array}{r}
\sum_{m \in \mathcal{S}} R_{m} \leq \log \operatorname{det}\left(\mathbf{I}_{N}+\frac{P_{m} v_{m}^{2}}{N_{0}} \mathbf{h}_{\mathcal{S}} \mathbf{h}_{\mathcal{S}}^{\mathrm{H}}\right), \\
\text { for each } \mathcal{S} \subset\{1, \ldots, M\},
\end{array}
$$

where $\mathbf{h}_{\mathcal{S}}$ contains the corresponding columns within $\mathbf{H}$. Since SIC based MMSE detection is used in this model, the above capacity region is achievable [13]. For example, if there are only two UAVs, the capacity region is drawn from (15) using the method introduced in [13] and shown in Fig.2, where $R_{1}=$ $\log \left(1+\frac{P_{m} v_{m}^{2} \mathbf{h}_{1}^{\mathrm{H}} \mathbf{h}_{1}}{N_{0}}\right), R_{2}=\log \left(1+\frac{P_{m} v_{m}^{2} \mathbf{h}_{2}^{\mathrm{H}} \mathbf{h}_{2}}{N_{0}}\right)$ and $R_{1}+R_{2}=$ $\mathcal{I}$ given by (3). The proposed model can thus theoretically support the maximum data rate; achieving corner points $C_{1}$ and $C_{2}$.

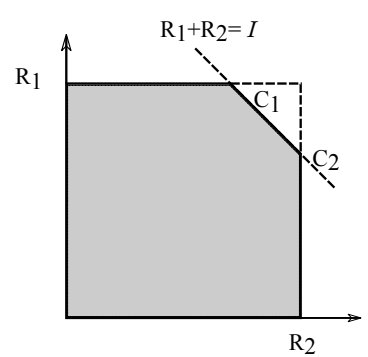

Fig. 2. Capacity region of 2 UAVs with maximum data rates $R_{1}$ and $R_{2}$.

From the analysis of average data rate, we can see that a well diversified and less correlated channel matrix, e.g. $\mathbf{H}$, is very important to the average data rate. This provides an insight of how to arrange the UAVs at rendezvous when they are connected to the relay. Intuitively the UAVs should be well separated to create a well-conditioned $\mathbf{H}$ but also within a certain physical distance so that the channels are strong enough to support the required minimum data rate.

\section{Optimization}

In this subsection we examine the optimization to minimize the maximum communication time of UAVs, given different amount of data at each UAV. As such the relay can use less time at each rendezvous and transport the data to station more frequently, and for the UAV-team, a reduction in waiting time provides more opportunity for sensing.

Suppose the $m$ th UAV currently has $D_{m}(m=1, \ldots, M)$ bits of data to be transmitted, where $D_{m}$ is the total data bits after framing and other preparation. From the previous model description, the instant data rate supported by the equivalent channel is given by

$$
\mathcal{E}_{m}=\log \left(1+\frac{P_{m} v_{m}^{2} \lambda_{m}}{N_{0}}\right)
$$

Generally the time required for data transmission can be calculated as follows,

$$
T_{m}=\frac{D_{m}}{\mathcal{E}_{m} * B_{m}}
$$

where $B_{m}$ is the bandwidth assigned to $U_{m}$. Thus the optimization strategy is defined

$$
\begin{array}{ll}
\operatorname{minimize} & \max \left\{T_{m}\right\}, m=1, \ldots, M \\
\text { subject to } & P_{\text {min }} \leq v_{m}^{2} P_{m} \leq P_{T},
\end{array}
$$

where $P_{\min }$ and $P_{T}$ are the minimum and maximum transmitting power.

The solution to the above optimization is given below,

1) UAVs inform the relay its amount of data to transfer, $D_{m}(m=1, \ldots, M)$ using minimum transmitting power $P_{\text {min }}$.

2) Relay estimates the channel matrix $\mathbf{H}$, calculates the average data rates, and then broadcasts them and the vector $\mathbf{D}=\left\{D_{1}, \ldots, D_{M}\right\}^{\mathrm{T}}$ to the whole team;

3) $U_{k}$ (with the largest payload and hence longest transfer time) increases its transmitting power to $P_{T}$ and the others adjust their power adaptively using the precoding matrix $\mathbf{V}(n)$, where $v_{i}, i=1, \ldots, M, i \neq k$ is calculated from (16) and (17); thus aiming for simultaneous completion of data transfers to the relay.

Fig. 3 shows the effect of optimization, where the channels are of IID Rician fading with $K=10$. The noise is set as AWGN and default SNR is 20dB. The amount of data collected by each user is drawn from a random Gaussian distribution $N(1000,500)$. Each UAV uses a bandwidth of $1 H z$ and can adjust its transmitting power within the allowed budget of $[-10 \mathrm{~dB}, 10 \mathrm{~dB}]$ (the adjusting factor). The optimization process finds appropriate transmission powers for all UAVs in order to complete data transmission simultaneously. For example, with all UAVs at $0 \mathrm{db}, \mathrm{UAV}-1$ would require 368 seconds to transfer its data, greater than all others. After optimization UAV-1 increases its power to maximum, with other UAVs making corresponding decreases, the team finishes transmitting to the relay simultaneously after 184 seconds, achieving power savings and improved team efficiency.

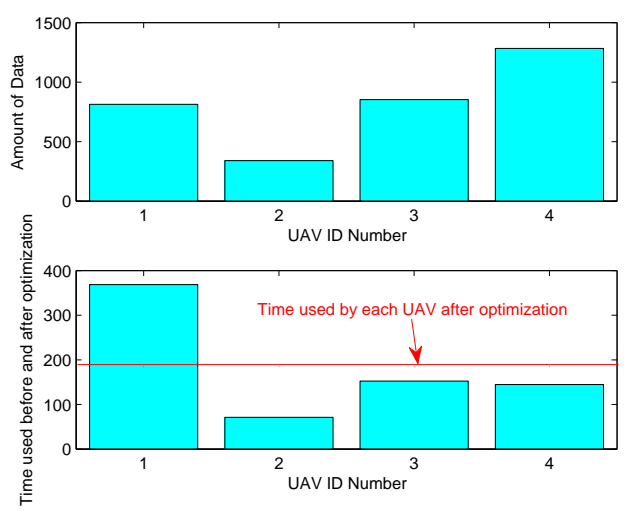

Fig. 3. The amount of data on each UAV and the corresponding time used by each one before and after optimization.

\section{Simulation EXPERIMENTS}

In order to assess the performance of the relay approach, the UAV deployment strategy has been implemented in a 
discrete time simulation. UAVs are modelled as quad-rotor rotor-craft with basic kinematic constraints, limited communication power and downward facing sensing capability for searchers. Each UAV models the ground area using a gridbased representation with a square considered as perceived when a sensor is passed over it at the appropriate altitude. The search environment is modelled from real terrain data from the UK Ordnance Survey data set with ground height accuracy of $5 \mathrm{~m}$ at a lateral resolution of $10 \mathrm{~m}$. All UAVs respect a minimum altitude of $20 \mathrm{~m}$ above ground level, using a standard A-star path planning algorithm for navigation.

The scenario consists of a fixed base-station and defined region for the team to search, see Fig. 4. A group of 4 searchers are deployed from base to the remote region with a single relay UAV responsible for providing a delay tolerant link between team and base using a high degree of mobility. Rendezvous meetings occur at the centre of the search area, with the timing arranged at the previous occurrence. Duration of each rendezvous is dependent on the time taken for all searchers to transfer the newly acquired data to the relay. This is considered to be proportional to the size of this data and hence the amount of search activity since the previous meeting. Each searching UAV selects a small area to search based on its current map and the provided terrain information. After generating a previously unvisited frontier area that can be sensed at a constant flight altitude, the UAV then searches this area with a sweep pattern, visiting every grid location within bounds.

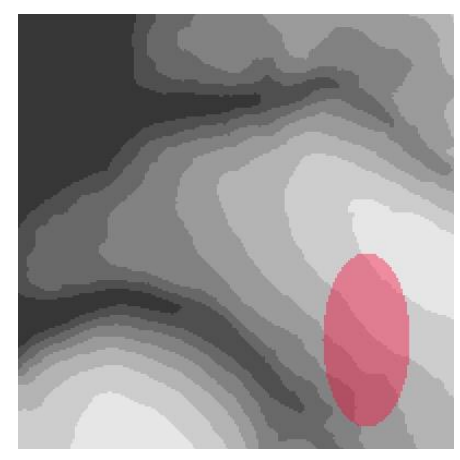

Fig. 4. A typical deployment environment, with terrain shown by shading (higher ground is brighter), an elliptical region to be searched by the team at the lower right (in red) and base station at upper left.

The information gathered during a typical deployment is shown in Fig. 5. The search team gathers new information at a consistent rate between rendezvous with the relay. The base station is periodically updated when, after a rendezvous, the relayer provides a batch of new data. The frequency of these updates depends on the travel time between base and meeting location and the duration of the data transfer. For this example a correctly configured relay communication is assumed, allowing all searcher UAVs to complete data transmission simultaneously. The approximate length of each rendezvous is shown by the periods of no increase in team information, as search operations have paused. As shown in Section III-D, this duration is proportional to the time consumed by the UAV with worst wireless link at this rendezvous.

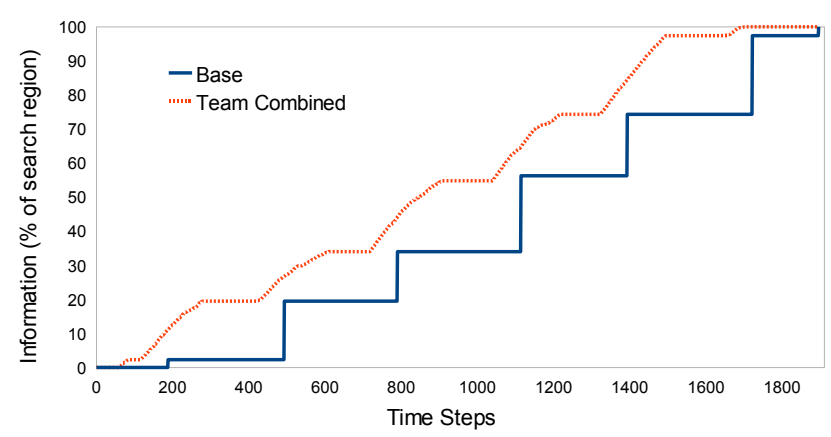

Fig. 5. Information gathered during search operations as a percentage of the designated search region, for both the base station and the entire team combined.

To examine the effectiveness of deploying a specific relay UAV, a comparison can be made with a team comprising completely of searching UAVs, with each searcher physically transporting new data to base on reaching a threshold. To make a fair comparison it is important to set an appropriate limit for this data cache: too low will artificially restrict the search rate and too high results in long periods where the base station receives no new information. To overcome this difficulty, each simulation is tuned to mirror the delay in freshly sensed information reaching base in an equivalent relay/rendezvous deployment. The new data threshold for searchers being set equals to the average data gathered by searchers between rendezvous when a relay is in use. This must be done for each scenario as the search rate and rendezvous periods depend on the spatial relationship between the base station and search region. In a practical non-relay deployment this would be configured to determine the balance between search rate and acceptable delay in receiving new information at base. The transfer duration of data from each searcher to the base station is considered proportional to the data size; each searcher is released when its individual transmission is completed. It is assumed that a fixed ground-based station has sufficient capacity to allow all searchers being connected simultaneously.

A comparison of the search efficiency of relay and non-relay search teams is shown in Fig. 6; including a relay-enabled team of 5 UAVs (1 relay, 4 searchers), a team of 4 searchers and a team of 5 searchers. The latter is included to examine the case where we have a limited number of vehicles and can choose how to configure them, e.g. as either relays or searchers. The plot shows the combined information gathered by the search team. These results were generated from taking averages of 10 simulations for each team configuration, using a constant size search region at a pre-defined set locations on the terrain map (similar to that shown in Fig. 4). In the initial phase the search-only teams gather data at a higher rate as they do not pause for an early rendezvous. However, the relayenabled team is able to achieve an increased search efficiency, covering the area more quickly than teams with either 4 or 5 
searchers. In these simulations the search region is relatively small (approximately $2.5 \mathrm{~km}^{2}$ ) in comparison to the application domain requirements. With an increased search area, the relay method would be expected to offer an even greater advantage in search efficiency.

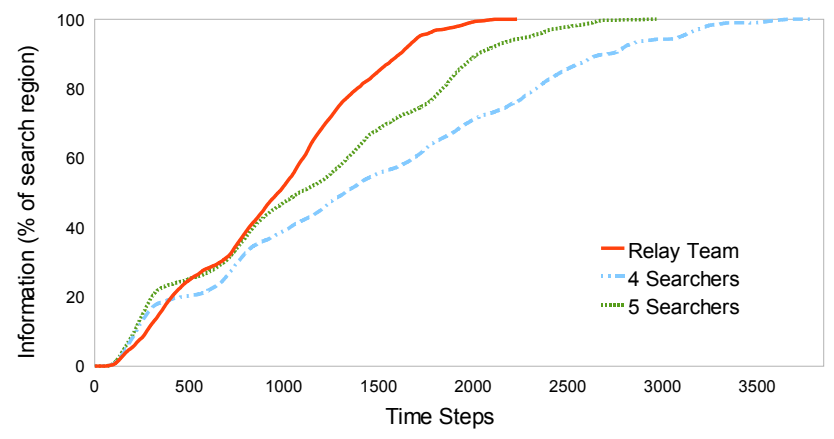

Fig. 6. A comparison of the relay approach with teams containing only searching UAVs. Data generated by averaging over 10 runs with differently located search regions.

\section{Discussion AND Future Work}

One advantage of the proposed model is its support of flexible number of UAVs in simultaneous connection as long as the number is not more than antennas on the relay: $M \leq N$. Thus a variation in team size, such as vehicle failure or malfunction, does not require a reconstruction of the whole network model. However, it will affect the transmission time of each UAV. Less UAVs within a group leads to less cochannel interference, thereby a reduction in the time taken to transfer the same amount of data for each participator.

The wireless channel often suffers deep fading [12], thus the outage probability is as important as the average data rate. In our future work, both the two metrics will be studied and tested. Besides, in this work we assume the deployment of a single relay and the combined travel and data exchange time does not cause an unacceptable delay. An obvious extension would use multiple relays to reduce the latency of data collection to availability at base. By refining this approach, the balance between search rate and data freshness at base can be tuned to meet an operational requirement.

This research has been supported by the Sensing Unmanned Autonomous Aerial VEhicles (SUAAVE) project, in which we hope to test swarms of UAVs in real search and rescue deployments [17]. The proposed model will be used as the backbone of the communication network and its suitability and efficiency will be tested in practice.

\section{CONCLUSION}

In this paper, we focus on the communication provision to support wilderness search and rescue. A team of UAVs are deployed to collect information with the support of a dedicated relay playing the role of data mule between them and base station. Theoretical analysis and experimental simulation have shown its suitability for scenarios with considerable distance between a base station and the search region of interest. The results demonstrate that it can be beneficial to allocate a vehicle as a designated relay instead of an additional searcher and achieve an overall increased search efficiency. In particular this method takes advantage of the excellent mobility and high levels of autonomy becoming available in unmanned autonomous vehicles by removing the requirement to maintain permanent communication links. This work is suitable for application in a range of additional cooperative sensing scenarios, including environmental monitoring and disaster response.

\section{REFERENCES}

[1] M. A. Goodrich, J. L. Cooper, J. A. Adams, C. Humphrey, R. Zeeman, and B. G. Buss, "Using a mini-UAV to support wilderness search and rescue: Practices for human-robot teaming," in Proceedings of the IEEE International Conference on Safety, Security and Rescue Robotics, 2007, pp. $1-6$.

[2] S. Waharte and N. Trigoni, "Supporting search and rescue operations with UAVs," in Proceedings of the 2010 International Conference on Emerging Security Technologies, ser. EST '10. Washington, DC, USA: IEEE Computer Society, 2010, pp. 142-147.

[3] W. Teacy, J. Nie, S. McClean, and G. Parr, "Maintaining connectivity in uav swarm sensing," in GLOBECOM Workshops (GC Wkshps), 2010 IEEE, dec. 2010, pp. $1771-1776$.

[4] M. A. Hsieh, A. Cowley, V. Kumar, and C. J. Taylor, "Maintaining network connectivity and performance in robot teams," Journal of Field Robotics, vol. 25, no. 1, pp. 111-131, 2008.

[5] P. Olsson, J. Kvarnstrom, P. Doherty, O. Burdakov, and K. Holmberg, "Generating uav communication networks for monitoring and surveillance," in Control Automation Robotics Vision (ICARCV), 2010 11th International Conference on, dec. 2010, pp. $1070-1077$.

[6] O. Hammouri and M. Matalgah, "Voronoi path planning technique for recovering communication in uavs," in Computer Systems and Applications, 2008. AICCSA 2008. IEEE/ACS International Conference on, 31 2008-april 4 2008, pp. 403 -406.

[7] S. Poduri and G. S. Sukhatme, "Constrained coverage for mobile sensor networks," in Proceedings of the IEEE International Conference on Robotics and Automation, 2004, pp. 165-172.

[8] Y. Ben-Asher, S. Feldman, P. Gurfil, and M. Feldman, "Distributed decision and control for cooperative uavs using ad hoc communication," Control Systems Technology, IEEE Transactions on, vol. 16, no. 3, pp. $511-516$, may 2008.

[9] B. Yamauchi, "Frontier-based exploration using multiple robots," in AGENTS '98: Proceedings of the second international conference on Autonomous agents. New York, NY, USA: ACM, 1998, pp. 47-53.

[10] J. de Hoog, S. Cameron, and A. Visser, "Selection of rendezvous points for multi-robot exploration in dynamic environments," in Workshop on Agents in Realtime and Dynamic Environments, International Conference on Autonomous Agents and Multi-Agent Systems (AAMAS), May 2010.

[11] N. Roy and G. Dudek, "Collaborative robot exploration and rendezvous: Algorithms, performance bounds and observations," $A u$ tonomous Robots, vol. 11, pp. 117-136, 2000.

[12] J. G. Proakis, Digital communications, 4th ed. New York: McGrawHill, Inc., 2001.

[13] D. Tse and P. Viswanath, Fundamentals of wireless communication. New York, NY, USA: Cambridge University Press, 2005.

[14] M. K. Simon and M.-S. Alouini, Digital Communication over Fading Channels (Wiley Series in Telecommunications and Signal Processing). Wiley-IEEE Press, Dec. 2004

[15] W.-Y. Shin, S.-Y. Chung, and Y. Lee, "Diversity-multiplexing tradeoff and outage performance for rician mimo channels," Information Theory, IEEE Transactions on, vol. 54, no. 3, pp. 1186 -1196, march 2008.

[16] M. Abramowitz and I. A. Stegun, Handbook of Mathematical Functions with Formulas, Graphs, and Mathematical Tables, 9th ed. New York: Dover, 1964.

[17] S. Cameron, S. Hailes, S. McClean, and et al., "Suaave: Combining aerial robots and wireless networking," in SUAAVE. University of Oxford, University College London, University of Ulster, feb. 2010, pp. 1 - 14. [Online]. Available: http://www.cs.ox.ac.uk/files/3070/bristol.pdf 